\title{
Hipertensão arterial no idoso saudável e no idoso frágil: uma revisão narrativa
}

\author{
Eduardo M. Costa ${ }^{1 *}$ Roberto A. Lourenço ${ }^{2}$
}

\begin{abstract}
Resumo
Introdução: Sabe-se que, com o envelhecimento populacional, a prevalência de doenças crônicas, como a hipertensão arterial (HA), aumenta. Além disso, a fisiologia do envelhecimento e de comorbidades a ele associadas faz com que seja necessário rever pontos de corte para definição de hipertensão no idoso, principalmente nos mais frágeis. Objetivo: Descrever, por meio de uma revisão narrativa, as recomendações para o tratamento da HA no paciente idoso e, especialmente, no frágil. Métodos: Revisão bibliográfica referente à HA no idoso, em bases de dados de pesquisa on-line. Resultados: Foram compiladas diretrizes nacionais, internacionais, além de artigos originais e de revisão sobre o assunto. Observou-se que não há um consenso quanto ao tratamento da HA, que este tratamento deve ser individualizado e que os efeitos colaterais das medicações precisam ser levados em consideração. Conclusão: Concluímos que os efeitos colaterais e interações entre drogas devem ser considerados na escolha da medicação para HA. Entende-se que a hipotensão pode predispor a efeitos indesejados como quedas e sonolência, por estes motivos, tanto a referência quanto o alvo para o nível tensional são maiores do que na população mais jovem.
\end{abstract}

Descritores: Hipertensão arterial; Idoso; Fragilidade.

\section{Abstract \\ Arterial hypertension in the healthy and in the fragile elderly: a narrative review}

Introduction: With the aging population, the prevalence of chronic diseases, such as arterial hypertension (AH), is increasing. In addition, the physiology of aging and other associated comorbidities makes it necessary to review cutoff points for the definition of hypertension in the elderly, especially the more fragile ones. Objective: To describe, through a narrative review, recommendations for the treatment of $\mathrm{AH}$ in the elderly, especially in the fragile ones. Methods: Bibliographic review of the topic of systemic arterial hypertension in the elderly, in an online search database. Results: National, international guidelines were compiled, as well as original and review articles on the subject. It was observed that there is no consensus regarding the treatment of $\mathrm{AH}$, that this treatment should be individualized and that the side effects of the medications need to be taken into account. Conclusions: In terms of choice of medication, the side effects and interactions between drugs must be considered in the AH treatment. It is understood that hypotension may predispose to unwanted effects such as falls and drowsiness, for these reasons, both the reference and the target for the tensional level are higher than in the younger population.

Keywords: Arterial hypertension; Elderly; Frailty.

\author{
1. Serviço de Geriatria Professor Mario A. Sayeg. Policlínica Piquet \\ Carneiro. Universidade do Estado do Rio de Janeiro, Rio de Janeiro, \\ RJ, Brasil. \\ 2. Departamento de Medicina Interna. Faculdade de Ciências \\ Médicas. Universidade do Estado do Rio de Janeiro, Rio de Janeiro, \\ RJ, Brasil.
}

\author{
*Endereço para correspondência: \\ Laboratório de Pesquisa em Envelhecimento Humano \\ GeronLab-UERJ \\ Av. Marechal Rondon 381, 20 andar \\ Rio de Janeiro, RJ. Brasil. CEP: 20950-000. \\ E-mail: eduardomagalhaes88@yahoo.com.br
}

Revista HUPE, Rio de Janeiro, 2017;16(1):37-43

doi: $10.12957 /$ rhupe.2017.33267

Recebido em 26/01/2018. Aprovado em 01/03/2018.

\section{Resumen}

Hipertension arterial en el anciano saludable y en el anciano frágile: una revisión

Introducción: Se sabe que con el envejecimiento poblacional la prevalencia de enfermedades crónicas, como la hipertensión arterial (HA) aumenta. Además, la fisiología del envejecimiento y otras comorbilidades asociadas hace que sea necesario revisar puntos de corte para definir la hipertensión en el anciano, principalmente en los más frágiles. Objetivo: Describir por medio de una revisión narrativa las recomendaciones para el tratamiento de la HA en el anciano, sobre todo en el anciano frágil. Métodos: Revisión bibliográfica del tema hipertensión arterial sistémica en el anciano, en una base de datos de investigación en línea. Resultados: Se recopilaron directrices nacionales, internacionales, además de artículos originales y de revisión sobre el tema. Se observó que no hay consenso en cuanto al tratamiento de la HA, que este tratamiento debe ser individualizado y que los efectos secundarios de los medicamentos deben ser tenidos en cuenta. Conclusiónes: En la elección de la medicación se deben tomar en consideración los efectos secundarios e interacciones entre drogas. Se entiende que la hipotensión puede predisponer a efectos no deseados como caídas y somnolencia, por estas razones, tanto la referencia y el objetivo del nivel tensional son más altos que en la población más joven.

Palabras clave: Hipertensión arterial; Ancianos; Fragilidad. 


\section{Artigo de revisão}

\section{Introdução}

A hipertensão arterial (HA) é a doença crônica não transmissível mais prevalente na população acima de 65 anos. Estima-se que nesta faixa etária sua prevalência ultrapasse $60 \%$. Além disso, esta condição vem associada a uma série de outras desordens comuns na terceira idade, tais como doença cardiovascular, doença renal crônica e síndrome demencial., ${ }^{1,2}$ Por este motivo sua identificação e tratamento adequados são extremamente importantes.

O estudo de Framingham mostrou, após 34 anos de acompanhamento, que os indivíduos com pressão arterial (PA) mais elevada apresentavam duas a quatro vezes mais risco de evoluir com insuficiência cardíaca. Além disso, apontou que 90\% dos indivíduos com PA normal até os 55 anos desenvolverão HA no decorrer da vida. ${ }^{3}$

Sabe-se que a HA, juntamente com outros fatores de risco, como diabetes mellitus, tabagismo e dislipidemia, aumenta consideravelmente o risco de eventos cardiovasculares, como infarto agudo do miocárdio e acidente vascular encefálico. ${ }^{2}$

No Brasil, estima-se que cerca de um terço dos óbitos são causados por doenças do sistema circulatório, como as duas últimas citadas acima. Desta maneira, fica evidente a necessidade de tratar a HA a fim de se evitarem as complicações que geram grande morbimortalidade na população idosa. ${ }^{1}$

Com o avançar da idade, ocorrem alterações na microarquitetura da parede dos vasos sanguíneos, levando a um enrijecimento arterial, principal mecanismo relacionado à elevação da PA associada ao envelhecimento. Como consequência, há perda da distensibilidade e da elasticidade, principalmente dos grandes vasos, como a artéria aorta, e consequente aumento da onda de pulso. ${ }^{2}$ Estas alterações podem levar a algumas alterações próprias do envelhecimento, como aumento da frequência do hiato auscultatório, além de uma pressão diastólica mantida normal ou até mais baixa. O idoso tende a apresentar variações na PA durante o dia, o que torna a monitorização ambulatorial da pressão arterial (MAPA) uma ferramenta bastante útil., ${ }^{1,2}$

Outras peculiaridades desta população precisam ser conhecidas. A pseudo-hipertensão está associada ao processo aterosclerótico, e consiste na permanência da artéria radial palpável, mesmo após a insuflação do manguito $30 \mathrm{mmHg}$ acima do desaparecimento do pulso radial, conhecida como manobra de Osler. Além desta, outras condições influenciam na PA do idoso, como a maior prevalência de arritmias cardíacas, principalmente a fibrilação atrial, e a hipotensão ortostática. Esta última é definida como a redução igual ou superior a $20 \mathrm{mmHg}$ e $10 \mathrm{~mm} \mathrm{Hg}$, na PA sistólica (PAS) e diastólica (PAD), respectivamente, quando comparadas nas posições sentada e em pé, com um intervalo de 3 minutos entre as medidas. ${ }^{2}$

Ainda não há um consenso sobre os valores normais de referência da PA para a população idosa, já que existem poucos estudos que incluem os muito idosos, ou seja, aqueles com mais de 80 anos de vida. Por esta razão, o manejo da HA nesta população deve ser individualizado e pautado no status funcional prévio do paciente. O objetivo desta revisão é avaliar o que há de recomendações e perspectivas para o tratamento de HA na terceira idade.

No que concerne o diagnóstico da HAS, além da aferição da PA em mais de uma posição, como descrito anteriormente, é importante lembrar que a medida deve ser repetida mais de uma vez, tendo em vista que muitos pacientes chegam ansiosos à consulta. Outros fatores relevantes são a ingesta de bebida alcóolica e café, realização de atividade física e o não esvaziamento vesical antes da consulta, fatores que podem elevar a PA. ${ }^{2,4}$

Além da aferição ambulatorial, devem-se considerar medições domiciliares e métodos complementares (MAPA), a fim de afastar erros diagnósticos como a hipertensão do jaleco branco e a hipertensão mascarada. Quando o diagnóstico é feito no ambulatório, recomendam-se pelo menos duas aferições da PA por consulta, em pelo menos duas consultas realizadas em dias diferentes. $1,4,5$

Segundo a $7^{a}$ Diretriz Brasileira de Hipertensão, ${ }^{2}$ recomenda-se o tratamento farmacológico para valores de PAS acima de $140 \mathrm{mmHg}$; entretanto, nos indivíduos acima de 80 anos este valor se eleva para $160 \mathrm{mmHg}$. Já o 8o Joint National Committee ${ }^{5}$ recomenda iniciar a terapia medicamentosa para valores de PAS acima de $150 \mathrm{mmHg}$ e PAD acima de $90 \mathrm{mmHg}$, tendo valores abaixo destes como meta. Já a diretriz europeia ${ }^{4}$ recomenda tratamento para valores de PAS acima de 160 mmHg. (Tabela 1)

Lesões de órgãos-alvo também têm de ser sempre pesquisadas em pacientes hipertensos, com o objetivo de avaliar o impacto sobre o organismo e o tempo de anormalidade dos níveis elevados de PA. Para isto, deve-se realizar exame de fundo de olho, além de laboratório com hemograma completo, eletrólitos, função renal e lipidograma, elementos anormais de sedimentação 
(EAS) e ecocardiograma., ${ }^{1,2}$

Segundo a $7^{\mathrm{a}}$ Diretriz Brasileira, a HA em indivíduos adultos deve ser classificada em função dos níveis tensionais médios obtidos durante a avaliação inicial e/ou durante o seguimento.(Tabela 3) É importante ressaltar que quando a PAS e a PAD situam-se em categorias diferentes, a maior deve ser utilizada para classificação; ainda, considera-se o diagnóstico de HA isolada se a PAS $\geq 140 \mathrm{~mm} \mathrm{Hg}$ e $\mathrm{PAD} \leq 90 \mathrm{~mm} \mathrm{Hg}$, devendo a mesma ser classificada nos estágios 1, 2 e 3, seguindo os níveis sistólicos expressos na tabela $2 .^{2}$

\section{Métodos}

Estudo de revisão narrativa, realizado com busca de artigos originais, de revisão, de livros textos, diretrizes nacionais e internacionais sobre a hipertensão arterial no idoso. Para a busca de artigos originais e de revisão usou-se banco de dados eletrônicos Pubmed e Scielo. Foram pesquisadas as palavras-chave: "Hipertensão Arterial", "Idoso "Hipertensão "Frágil", "Fragilidade", "Arterial Hypertension" "Elderly" e "Frailty".

\section{Resultados}

Para este estudo foram selecionados 1 livro texto de geriatria, 3 diretrizes sobre o tratamento da HA e 22 artigos acerca do tema. Este material será analisado e discutido a seguir.

\section{Tratamento da HA}

A decisão quanto ao início do tratamento farmacológico na HA é sempre individualizada. Deve-se levar em consideração o status funcional do idoso, perfil de aderência, lesões em órgãos-alvo, além, evidentemente, da medida de PA. Pode-se recomendar inicialmente mudanças no estilo de vida (MEV), com intuito de poupar o uso de medicamentos e seus efeitos colaterais em uma população que já convive com a polifarmácia.

Estudos mostram o benefício da redução da PA quando a PAS for maior que $160 \mathrm{mmHg}$ e tiver seu valor reduzido para abaixo de $150 \mathrm{mmHg}$. Em idosos abaixo de 80 anos, com bom status funcional, pode-se reduzir o valor para abaixo de $140 \mathrm{mmHg}$. Manter a PAS abaixo de 150 mmHg em pacientes acima de 80 anos, também com funcionalidade preservada, tem efeito protetor de eventos cardiovasculares adversos. ${ }^{1,2,6}$

\section{Mudanças no estilo de vida}

Independente do estágio da HA, as MEV são sempre benéficas. Nos estágios mais leves, elas podem ser responsáveis pelo controle pressórico adequado. Para isto, uma abordagem multidisciplinar com nutricionistas, profissionais de educação física e fisioterapeutas é sempre recomendada. ${ }^{1}$

As principais atividades que compõem as MEV consistem em redução do peso corporal, redução na ingestão de sódio, redução do consumo de bebidas alcóolicas e prática de exercícios físicos. Sabe-se que muitos idosos apresentam limitações físicas que impedem esta última, entretanto as outras medidas podem ser tomadas. ${ }^{2}$

Além da alteração de hábitos de vida, o controle da polifarmácia é imprescindível. Muitos idosos fazem uso de medicações capazes de elevar a PA, como os anti-inflamatórios não esteroides, os anti-histamínicos, os antidepressivos tricíclicos, os hormônios tireoidianos em altas doses, os antiácidos ricos em sódio e moderadores de apetite.

\section{Farmacológico}

O tratamento farmacológico visa controlar a PA, além de reduzir as complicações decorrentes desta patologia. Esta abordagem deve ser individualizada, levando em consideração as comorbidades de cada paciente. Algumas recomendações devem ser seguidas na escolha da droga anti-hipertensiva a ser utilizada, são elas: ${ }^{2}$

- ter demonstrado a capacidade de reduzir a morbimortalidade cardiovascular;

- ser eficaz por via oral;

- ser bem tolerada;

- poder ser usada no menor número de tomadas por dia;

- ser iniciada com as menores doses efetivas;

- poder ser usada em associação;

Tabela 1. Valores de referência segundo as diretrizes brasileira, norte-americana e europeia

\begin{tabular}{|c|c|c|c|}
\hline & 7ª Diretriz Brasileira (2016) & 8 JOINT (2014) & Diretriz Europeia (2013) \\
\hline $\begin{array}{l}\text { Indicação de tratamento } \\
\text { farmacológico no idoso } \\
\text { (> } 60 \text { anos) }\end{array}$ & $\begin{array}{l}\text { - PAS } \geq 140 \mathrm{mmHg} \\
\text { - PAS } \geq 160 \mathrm{mmHg} \text { (se maior de } \\
80 \text { anos) }\end{array}$ & $\begin{array}{l}-\mathrm{PAS} \geq 150 \mathrm{mmHg} \\
-\mathrm{PAD} \geq 90 \mathrm{mmHg}\end{array}$ & - PAS $\geq 160 \mathrm{mmHg}$ \\
\hline
\end{tabular}

Fonte: O autor (2018). 


\section{Artigo de revisão}

Tabela 2. Classificação da pressão arterial sistêmica segundo estágios de gravidade

\begin{tabular}{c|c|c|}
\hline Classificação & PAS $(\mathrm{mmHg})$ & PAD $(\mathrm{mmHg})$ \\
\hline Normal & $\leq 120$ & $\leq 80$ \\
\hline Pré-hipertensão & $121-139$ & $81-99$ \\
\hline Hipertensão estágio 1 & $140-159$ & $90-99$ \\
\hline Hipertensão estágio 2 & $160-179$ & $100-109$ \\
\hline Hipertensão estágio 3 & $\geq 180$ & $\geq 110$ \\
\hline
\end{tabular}

Fonte: Adaptado da 7ạ Diretriz Brasileira de HA (2016).
- ser utilizada por um período mínimo de quatro semanas, antes de modificações, salvo em situações especiais;

- ter controle de qualidade em sua produção.

Na escolha do anti-hipertensivo a ser utilizado a preferência será sempre pelos que tenham maior efeito protetor cardiovascular e apresente menos efeitos colaterais. Considerando-se a polifarmácia, muito comum nesta faixa etária, deve-se ficar atento para as possíveis interações medicamentosas. Na tabela 3 estão descritos os efeitos benéficos e adversos das principais classes

Tabela 3. Ações esperadas e efeitos adversos de medicações anti-hipertensivas

\begin{tabular}{|c|c|c|}
\hline Classe & Ação & Efeitos adversos \\
\hline Diuréticos & $\begin{array}{l}\text { - Diminui morbimortalidade cardiovascular } \\
\text { - Tiazídicos: maior tempo de ação, doses mais baixas } \\
\text { - De alça: insuficiência renal e edema } \\
\text { - Poupadores de potássio: associados aos outros diuréticos }\end{array}$ & $\begin{array}{l}\text { - Fraqueza } \\
\text { - Câimbras } \\
\text { - Piora incontinência urinária } \\
\text { - Disfunção erétil } \\
\text { - Hipopotassemia } \\
\text { - Hipomagnesemia } \\
\text { - Hiponatremia, hipovolemia } \\
\text { - Intolerância à glicose }\end{array}$ \\
\hline Agentes de ação central & $\begin{array}{l}\text { - Diminuição da atividade simpática } \\
\text { - Diminuição do reflexo dos barorreceptores }\end{array}$ & $\begin{array}{l}\text { - Hipotensão postural } \\
\text { - Boca seca } \\
\text { - Sonolência } \\
\text { - Disfunção erétil } \\
\text { - Cansaço }\end{array}$ \\
\hline Betabloqueadores & $\begin{array}{l}\text { - Diminuição do débito cardíaco } \\
\text { - Diminuição da secreção de renina }\end{array}$ & $\begin{array}{l}\text { - Bradiarritmias } \\
\text { - Broncoespasmo } \\
\text { - Depressão } \\
\text { - Disfunção erétil }\end{array}$ \\
\hline Alfabloqueadores & $\begin{array}{l}\text { - Diminuição da resistência vascular periférica } \\
\text { - Efeito muito discreto em monoterapia }\end{array}$ & $\begin{array}{l}\text { - Hipotensão } \\
\text { - Incontinência urinária }\end{array}$ \\
\hline Vasodilatadores diretos & $\begin{array}{l}\text { - Relaxam musculatura lisa arterial } \\
\text { - Reduz resistência vascular periférica }\end{array}$ & $\begin{array}{l}\text { - Anorexia, náuseas, vômitos } \\
\text { - Lupus like } \\
\text { - Cautela em doença arterial } \\
\text { coronariana } \\
\text { - Evitar em histórias de hemorragia } \\
\text { intracraniana e aneurisma de aorta }\end{array}$ \\
\hline $\begin{array}{l}\text { Bloqueadores do canal de } \\
\text { cálcio }\end{array}$ & $\begin{array}{l}\text { - Redução da resistência vascular periférica } \\
\text { - Boa opção para portadores de doença arterial coronariana } \\
\text { - Efeito vasodilatador }\end{array}$ & $\begin{array}{l}\text { - Edema de membros inferiores } \\
\text { - Cefaleia latejante } \\
\text { - Tonturas }\end{array}$ \\
\hline $\begin{array}{l}\text { Inibidores da enzima } \\
\text { conversora da angiotensina }\end{array}$ & $\begin{array}{l}\text { - Inibe ação vasoconstritora da angiotensina II, após bloqueio } \\
\text { da angiotensina I } \\
\text { - Boa opção para insuficiência cardíaca } \\
\text { - Retarda declínio da função renal na nefropatia diabética }\end{array}$ & $\begin{array}{l}\text { - Tosse seca } \\
\text { - Hiperpotassemia em pacientes } \\
\text { com doença renal } \\
\text { - Pode elevar ureia e creatinina }\end{array}$ \\
\hline $\begin{array}{l}\text { Bloqueadores de receptores } \\
\text { AT1 da angiotensina }\end{array}$ & $\begin{array}{l}\text { - Bloqueio direto da angiotensina II } \\
\text { - Bom em pacientes com alto risco de disfunção cardiovascular }\end{array}$ & - Exantemas \\
\hline
\end{tabular}


Eduardo M Costa, Roberto A Lourenço. • Hipertensão arterial no idoso saudável e no idoso frágil: uma revisão narrativa

Tabela 4. Classes, substâncias e posologia das principais drogas utilizadas no tratamento de hipertensão arterial

\begin{tabular}{|c|c|c|c|}
\hline \multirow{2}{*}{ Medicamentos } & \multicolumn{2}{|c|}{ Posologia diária (mg) } & \multirow{2}{*}{ Número de tomadas ao dia } \\
\hline & Mínima & Máxima & \\
\hline \multicolumn{4}{|l|}{ 1. Diuréticos tiazídicos } \\
\hline Clortalidona & 12,5 & 50 & 1 \\
\hline Hidroclorotiazida & 12,5 & 50 & 1 \\
\hline Indapamida & 1,5 & 5 & 1 \\
\hline \multicolumn{4}{|l|}{ 2. Diurético de alça } \\
\hline Furosemida & 20 & Variável & $1-2$ \\
\hline \multicolumn{4}{|c|}{ 3. Diuréticos poupadores de potássio } \\
\hline Espironolactona & 50 & 200 & $1-3$ \\
\hline \multicolumn{4}{|c|}{ 4. Inibidores Adrenérgicos } \\
\hline \multicolumn{4}{|l|}{ 4.1 Ação Central } \\
\hline Clonidina & 0,1 & 0,6 & $2-3$ \\
\hline \multicolumn{4}{|l|}{ 4.2 Alfabloqueadores } \\
\hline Doxazosina & 1 & 16 & 1 \\
\hline \multicolumn{4}{|l|}{ 4.3 Betabloqueadores } \\
\hline Atenolol & 25 & 100 & $1-2$ \\
\hline Bisoprolol & 2,5 & 10 & $1-2$ \\
\hline Carvedilol & 12,5 & 50 & 2 \\
\hline Metoprolol & 50 & 200 & $1-2$ \\
\hline Propranolol & 40 & 240 & $2-3$ \\
\hline \multicolumn{4}{|c|}{ 5. Vasodilatadores diretos } \\
\hline Hidralazina & 50 & 200 & $2-3$ \\
\hline \multicolumn{4}{|c|}{ 6. Antagonistas do canal de cálcio } \\
\hline Verapamil & 120 & 480 & $1-2$ \\
\hline Diltiazem & 60 & 360 & 3 \\
\hline Anlodipino & 2,5 & 10 & 1 \\
\hline Nifendipino & 20 & 60 & $2-3$ \\
\hline Nitrendipino & 10 & 40 & $1-2$ \\
\hline \multicolumn{4}{|c|}{ 7. Inibidores da Enzima Conversora da Angiotensina (IECA) } \\
\hline Captopril & 25 & 150 & $2-3$ \\
\hline Enalapril & 5 & 40 & $1-2$ \\
\hline Lisinopril & 5 & 20 & $1-2$ \\
\hline Ramipril & 2,5 & 10 & $1-2$ \\
\hline \multicolumn{4}{|c|}{ 8. Antagonistas do receptor de angiotensina II } \\
\hline Candesartana & 8 & 16 & 1 \\
\hline Irbesartana & 150 & 300 & 1 \\
\hline Losartana & 25 & 100 & 1 \\
\hline Olmesartana & 20 & 40 & 1 \\
\hline Valsartana & 40 & 320 & $1-2$ \\
\hline
\end{tabular}

Fonte: Adaptado de: Tratado de Geriatria e Gerontologia, 4a Edição (2016). 


\section{Artigo de revisão}

medicamentosas utilizadas.

Entendidas as classes de medicamentos anti-hipertensivos, agora se faz necessária a escolha do fármaco. Na tabela 4, listamos as principais drogas e sua posologia habitual. ${ }^{1}$

\section{HA nos idosos frágeis}

A fragilidade no idoso está relacionada à redução da reserva funcional de múltiplos sistemas fisiológicos. Como consequência, estes idosos apresentam um elevado risco de incapacidade e morte. ${ }^{7}$ Em um estudo realizado com idosos a partir de 65 anos a prevalência de fragilidade ficou em torno de $10 \% .^{8}$ Já em idosos acima de 85 anos, estima-se que até 50\% apresentem fragilidade. ${ }^{9}$ Sabe-se que os idosos frágeis apresentam níveis pressóricos mais elevados..$^{10}$ Ao mesmo tempo, esta população é a que possui maior risco de danos ao se baixar a pressão arterial.11

O estudo HYVET, mostrou que a fragilidade não reduziu o benefício que o tratamento da HA traz na prevenção a eventos cardiovasculares. ${ }^{12}$ Já o estudo SHEP diz que a evidência de redução de risco cardiovascular com o tratamento da HA desaparece em pacientes com limitação funcional. ${ }^{13,14}$ A diferença encontrada nestes dois estudos decorre, provavelmente, da divergência na definição de fragilidade empregada pelos autores. Observou-se também que a menor mortalidade em pacientes com capacidade funcional e cognitiva comprometidas foi encontrada com a medida da PA em 164 x $85 \mathrm{mmHg}$ e que participantes com PAS abaixo de 120 mmHg apresentavam maior risco relativo de morte que aqueles com PAS entre 160 e 179 mmHg. ${ }^{6}$ Outros estudos mostraram que reduzir a PA a níveis abaixo de $130 \mathrm{mmHg}$ e o uso de dois ou mais anti-hipertensivos também estão associados a maior mortalidade.15,16

Dessa maneira, preconiza-se que a abordagem do paciente frágil seja individualizada. Recomenda-se o início de terapia farmacológica para valores de PAS > $160 \mathrm{mmHg}$, com alvo de $150 \mathrm{mmHg}$. Caso os valores atinjam $130 \mathrm{mmHg}$ ou menos, deve-se considerar a desprescrição, o que pode levar a uma redução significativa da mortalidade segundo alguns estudos. ${ }^{17-22}$

Outro fator relevante em pacientes frágeis é a adesão ao tratamento medicamentoso. Acredita-se que quanto maior o grau de fragilidade, menor o cumprimento da prescrição. ${ }^{23,24}$ Sabe-se também que em idosos com grande comprometimento cognitivo o tratamento, além de não trazer benefícios, pode ainda piorar a cognição. ${ }^{25}$ Outro risco de se baixar a PA de idosos frágeis está no fato de alguns deles necessita- rem de níveis pressóricos mais elevados para manter a perfusão de alguns órgãos, dada a rigidez dos vasos por aterosclerose. $^{26}$

\section{Conclusão}

Considerando os fatos e estudos expostos neste artigo, podemos concluir que o tratamento da HA em idosos frágeis deve ser sempre individualizado. Sabe-se que valores de PAS abaixo de 130 mmHg estão relacionados com maior morbimortalidade nesta população. Além disso, estes pacientes apresentam menor aderência ao tratamento e são mais susceptíveis aos efeitos colaterais medicamentosos.

\section{Referências}

1. Campana EMG, Freitas EV, Brandão AA, et al. Hipertensão arterial no idoso. In: Freitas EV, Py L. Tratado de Geriatria e Gerontologia. 4a Edição. Rio de Janeiro: Guanabara Koogan; 2016. p. 839-60.

2. VII Diretrizes Brasileiras de Hipertensão. Arq Bras Cardiol. 2016;107(supl. 3).

3. Fox CS, Evans JC, Larson MG, et al. Temporal trends in coronary heart disease mortality and sudden cardiac death from 1950 to 1999: the Framingham Heart Study. Circulation. 2004;110:522-7.

4. 2013 ESH/ESC Guidelines for the management of arterial hypertension. The Task Force for the management of arterial hypertension of the European Society of Hypertension (ESH) and of the European Society of Cardiology (ESC). J Hypertens. 2013;31:1281-357.

5. 2014 Evidence based guideline for the management of high blood pressure in adults: Report from the panel members appointed to the Eighth Joint National Committee (JNC 8). JAMA. 2014;311(5):507-20.

6. Ogliari G, Westendorp R, Muller M, et al. Blood pressure and 10-year mortality risk in the milan geriatrics $75+$ cohort study: role of functional and cognitive status. Age Ageing. 2015;44:932-7.

7. Fried LP, Tangen CM, Walston J, et al. Frailty in older adults: evidence for a phenotype. J Gerontol A Biol Sci Med Sci. 2001;56(3):146-56.

8. Moreira VG, Lourenço RA. Prevalence and factors associated with frailty in an older population from the city of Rio de Janeiro, Brazil: the FIBRA-RJ Study. Clinics (SP). 2013;68(7)979-85.

9. Benetos A, Rossignol P, Cherubini A, et al. Polypharmacy in the aging patient: management of hypertension in octogenarians. JAMA. 2015;314:170-80.

10. Bastos-Barbosa RG, Ferriolli E, Coelho EB, et al. Association of frailty syndrome in the elderly with higher blood pressure and other cardiovascular risk factors. Am J Hypertens. 2012;25(11).

11. Odden M, Beilby P, Peralta C. Blood pressure in older adults: the importance of frailty. Curr Hypertens Rep. 2015;17(55).

12. Warwick J, Falaschetti E, Rockwood K, et al. No evidence that frailty modifies the positive impact of antihypertensive treatment in very elderly people: an investigation of the impact of frailty upon treatment effect in the hypertension in the very elderly trial (HYVET) study, a double-blind, placebo controlled study of antihypertensives in people with hypertension aged 80 and over. BMC Med. 2015;13:78. 
Eduardo M Costa, Roberto A Lourenço. • Hipertensão arterial no idoso saudável e no idoso frágil: uma revisão narrativa

13. Charlesworth C, Peralta C, Odden M. Functional status and antihypertensive therapy in older adults: a new perspective on old data. Am J Hypertens. 2016;29:690-5.

14. SHEP cooperative research group. Prevention of stroke by antihypertensive drug treatment in older persons with isolated systolic hypertension. Final results of the Systolic hypertension in the elderly program (SHEP). JAMA. 1991;265:3255-64.

15. Mancia G, Grassi G. Aggressive blood pressure lowering is dangerous: the J-curve: pro side of the argument. Hypertension. 2014;63:29-36.

16. Benetos A, Labat C, Rossignol P, et al. Treatment with multiple blood pressure medications, achieved blood pressure, and mortality in older nursing homeresidents: the PARTAGE study. JAMA Intern Med. 2015;175:989-95.

17. Benetos $A$, Rossignol $P$, Cherubini $A$, et al. Polypharmacy in the aging patient: management of hypertension in octogenarians. JAMA. 2015;314:170-80.

18. Bejan-Angoulvant T, Saadatian-Elahi M, Wright J, et al. Treatment of hypertension in patients 80 years and older: the lower the better? A meta-analysis of randomized controlled trials. J Hypertens. 2010;28:1366-72.

19. Page A, Clifford R, Potter K, et al. The feasibility and the effect of deprescribing in older adults on mortality and health: a sys- tematic review. Br J Clin Pharmacol. 2016;82:583-623.

20. Scott I, Hilmer S, Reeve E, et al. Reducing inappropriate polypharmacy the process of deprescribing. JAMA Intern Med. 2015;175:827-34.

21. Ferri C, Ferri L, Desideri G. Management of hypertension in the elderly and frail elderly. High Blood Press Cardiovasc Prev. 2017;24:1.

22. Ravindrarajah R, Hazra NC, Hamada S, et al. Systolic blood pressure trajectory, frailty and all-cause mortality over 80 years of age. Cohort study using electronic health records. Circulation. 2017;135(24):2357-68.

23. Chudiak A, Jankowska-Polanska B, Uchmanowicz I. Effect of frailty syndrome on treatment compliance in older hypertensive patients. Clin Interv Aging. May 2017:12.

24. Jankowska-Polanska A, Dudek K, Szymanska-Chabowska A, Uchmanowicz I. The influence of frailty syndrome on medication adherence among elderly patients with hypertension. Clinical Interventions in Aging. 2016;11:1781-90.

25. Ettehad D, Emdin CA, Kirin A, et al. Blood pressure lowering for prevention of cardiovascular disease and death: a systematic review and meta-analysis. Lancet. 2015;387:957-67.

26. Goodwin JS. Gait speed. An important vital sign in old age. Arch Intern Med. 2012;172:1168-9. 